\title{
Can Black Holes be Created at the Birth of the Universe ?
}

\author{
Zhong Chao Wu \\ Dept. of Physics \\ Beijing Normal University \\ Beijing 100875, China \\ (Gravity Essay)
}

\begin{abstract}
We study the quantum creation of black hole pairs in the (anti-)de Sitter space background. These black hole pairs in the Kerr-Newman family are created from constrained instantons. At the $W K B$ level, for the chargeless and nonrotating case, the relative creation probability is the exponential of (the negative of) the entropy of the universe. Also for the remaining cases of the family, the creation probability is the exponential of (the negative of) one quarter of the sum of the inner and outer black hole horizon areas. In the absence of a general no-boundary proposal for open universes, we treat the creations of the closed and the open universes in the same way.
\end{abstract}

PACS number(s): 98.80.Hw, 98.80.Bp, 04.60.Kz, 04.70.Dy

Keywords: quantum cosmology, constrained gravitational instanton, black hole creation

e-mail:wu@axp3g9.icra.it 
There are three ways of forming black hole in Nature. The first way is through the gravitational collapse of a massive body in astrophysics. In this scenario, the spacetime and matter content are treated classically. In general, the effect of the cosmological background is ignored. The second way originates from the quantum fluctuation of the matter content in the very early universe. Here the spacetime is again treated classically. The black hole formation is a result of the competing effects of the expansion of the universe and the gravitational attraction of the matter fluctuation. The third way is through the quantum creation of black holes in quantum cosmology, to which this paper is addressed. Here, both the spacetime and the matter content are quantized. This is the most dramatic type of black hole formation. Indeed, the black holes are essentially created from nothing at the same moment as the birth of the universe. Therefore, only black holes created this way are genuinely primordial.

It is believed that the Planckian era of the universe underwent an inflationary stage which was approximated by the de Sitter metric. In the Planckian stage, the potential of the scalar field behaves as an effective cosmological constant $\Lambda$. On the other hand, extended theories of supergravity in which the $O(N)$ group is gauged have the anti-de Sitter space as their ground or most symmetric state. Therefore, it is of great interest to study quantum creations of black holes in these backgrounds.

In the No-Boundary Universe, the wave function of a closed universe is defined as a path integral over all compact 4-metrics with matter fields [1]. The dominant contribution to the path integral is from the stationary action solution. At the $W K B$ level, the wave function can be approximated as $\Psi \approx e^{-I}$, where $I=I_{r}+i I_{i}$ is the complex action of the solution.

The imaginary part $I_{i}$ and real part $I_{r}$ of the action represent the Lorentzian and Euclidean evolutions in real time and imaginary time, respectively. The probability of a Lorentzian orbit remains constant during its evolution. One can identify the probability, not only as the probability of the universe created, but also as the probabilities for other Lorentzian universes obtained through an analytic continuation from it [2].

An instanton is defined as a stationary action orbit and satisfies the Einstein equation every- 
where. It was thought that, at the $W K B$ level, an instanton was the seed for the creation of the universe. Very recently, it was realized that this only applied to the case of creation with a stationary probability. Therefore, in order not to exclude many interesting phenomena and more realistic models from the study, one has to appeal to the concept of constrained instantons [3][4][5]. Constrained instantons are the orbits with an action that is stationary under some restriction. The restriction can be imposed on a spacelike 3-surface of the created Lorentzian universe. The restriction is that the 3-metric and matter content are given at the 3-surface. The relative creation probability from the instanton is the exponential of the negative of the real part of the instanton action.

One can begin with a complex solution to the Einstein equation and other field equations in the complex domain of spacetime coordinates. If an instanton exists at all, then it should be a compact singularity-free section of the solution. If there are singularities in the compact section, then, in general, the action of the section is not stationary. The action may only be stationary with respect to the variations under some restrictions mentioned above. We call this section a constrained gravitational instanton. To find the constrained instanton, one has to closely investigate the singularities. The stationary action condition is crucial to the validation of the $W K B$ approximation, which we use to investigate the problem of quantum creation of a black hole pair.

In contrast to the case for a closed universe, a general no-boundary proposal for the quantum state of an open universe has not been presented. However, one can use analytic continuation from a complex constrained instanton to obtain the $W K B$ approximation to the wave function for open universes with some kind of symmetry. At this level, both the open and closed creations of universes can be dealt with in the same way. For examples, The $S^{4}$ space model with $O(5)$ symmetry [6] and the $F L R W$ space model with $O(4)$ symmetry [2] have been investigated this way.

The constrained gravitational instantons for the pair creation of black holes in the (anti-)de Sitter space background can be obtained from the complex solutions of the Kerr-Newman-(anti-)de Sitter family [7]

$$
d s^{2}=\rho^{2}\left(\Delta_{r}^{-1} d r^{2}+\Delta_{\theta}^{-1} d \theta^{2}\right)+\rho^{-2} \Xi^{-2} \Delta_{\theta} \sin ^{2} \theta\left(a d t-\left(r^{2}+a^{2}\right) d \phi\right)^{2}-\rho^{-2} \Xi^{-2} \Delta_{r}\left(d t-a \sin ^{2} \theta d \phi\right)^{2},
$$

where

$$
\rho^{2}=r^{2}+a^{2} \cos ^{2} \theta
$$




$$
\begin{gathered}
\Delta_{r}=\left(r^{2}+a^{2}\right)\left(1-\Lambda r^{2} 3^{-1}\right)-2 m r+Q^{2}, \\
\Delta_{\theta}=1+\Lambda a^{2} 3^{-1} \cos ^{2} \theta, \\
\Xi=1+\Lambda a^{2} 3^{-1}
\end{gathered}
$$

and $m, m a$ and $Q$ are constants, representing mass, angular momentum, electric or magnetic charges. We shall not consider the dyonic case in the following. We shall respectively call the cases with de Sitter and anti-de Sitter backgrounds as closed and open models.

We use $r_{0}, r_{1}, r_{2}$ and $r_{3}$ to denote the four roots of $\Delta_{r}$. For the closed model with positive $\Lambda$, we assume all roots $r_{0}, r_{1}, r_{2}$ and $r_{3}$ are real and in ascending order. These roots are the negative, inner black hole, outer black hole and cosmological horizons, respectively. For the open model with negative $\Lambda$, at least two roots, say $r_{0}, r_{1}$, are complex conjugates, and we assume $r_{2}$ and $r_{3}$ are real. If this is the case, then $r_{2}$ and $r_{3}$ must be positive and can be identified as the inner and outer black hole horizons, respectively.

For the closed model [3], the constrained instanton is constructed from the metric (3) by setting $\tau=i t$. One makes two cuts at $\tau= \pm \Delta \tau / 2$ between the two horizons $r_{1}, r_{2}$ and glues them. The resultant manifold may have conical singularities at the two horizons. It has the $f_{1}$-fold cover around the horizon $r_{1}$ and the $f_{2}$-fold cover around the horizon $r_{2}$.

The Lorentzian metric for the created black hole pair is obtained through analytic continuation of the time coordinate from an imaginary value to a real value at the equator. The equator is two joint sections $\tau=$ consts. passing these horizons. It divides the instanton into two halves. We can impose the restriction that the 3 -geometry characterized by the parameters $m, a$ and $Q$ is given at the equator for the Kerr-Newman-de Sitter family. The parameter $\Delta \tau$ is the only degree of freedom left for the pasted manifold, since the field equation holds everywhere with the possible exception of these horizons. Thus, in order to check whether we get a stationary action solution for the given horizons, one only needs to see whether the above action is stationary with respect to this parameter. The equator where the quantum transition will occur has the topology $S^{2} \times S^{1}$.

The action due to the horizons is [3]

$$
I_{i, \text { horizon }}=-\frac{\pi\left(r_{i}^{2}+a^{2}\right)\left(1-f_{i}\right)}{\Xi} .(i=1,2)
$$


The action due to the volume is

$$
I_{v}=-\frac{\Delta \tau \Lambda}{6 \Xi^{2}}\left(r_{2}^{3}-r_{1}^{3}+a^{2}\left(r_{2}-r_{1}\right)\right) \pm \frac{\Delta \tau Q^{2}}{2 \Xi^{2}}\left(\frac{r_{1}}{r_{1}^{2}+a^{2}}-\frac{r_{2}}{r_{2}^{2}+a^{2}}\right),
$$

where $+(-)$ is for the magnetic (electric) case.

If one naively takes the exponential of the negative of half the total action, then the exponential is not identified as the wave function at the creation moment of the black hole pair. The physical reason is that what one can observe is only the angular differentiation, or the relative rotation of the two horizons. This situation is similar to the case of a Kerr black hole pair in the asymptotically flat background. There one can only measure the rotation of the black hole horizon from spatial infinity. To find the wave function for the given mass and angular momentum one has to make the Fourier transformation [3][8]

$$
\Psi\left(a, h_{i j}\right)=\frac{1}{2 \pi} \int_{-\infty}^{\infty} d \delta e^{i \delta J \Xi^{-2}} \Psi\left(\delta, h_{i j}\right),
$$

where $\delta$ is the relative rotation angle for the half time period $\Delta \tau / 2$, which is canonically conjugate to the angular momentum $J=m a$; and the factor $\Xi^{-2}$ is due to the time rescaling. The angle difference $\delta$ can be evaluated

$$
\delta=\int_{0}^{\Delta \tau / 2} d \tau\left(\Omega_{1}-\Omega_{2}\right)
$$

where the angular velocities at the horizons are $\Omega_{i}=a\left(r_{i}^{2}+a^{2}\right)^{-1}$.

In the magnetic case the vector potential determines the magnetic charge, which is the integral over the $S^{2}$ factor. However, in the electric case, one can only fix the integral

$$
\omega=\int A
$$

where the integral is around the $S^{1}$ direction, and $A$ is the vector potential of the electric field [3]. So, what one obtains in this way is $\Psi\left(\omega, a, h_{i j}\right)$. However, one can get the wave function $\Psi\left(Q, a, h_{i j}\right)$ for a given electric charge through the Fourier transformation [3][8][9][10]

$$
\Psi\left(Q, a, h_{i j}\right)=\frac{1}{2 \pi} \int_{-\infty}^{\infty} d \omega e^{i \omega Q} \Psi\left(\omega, a, h_{i j}\right)
$$

The Fourier transformations (8) and (11) for the angular momentum and the electric charge are equivalent to adding extra terms into the action for the constrained instanton, and then the total 
action becomes [3]

$$
I=-\pi\left(r_{1}^{2}+a^{2}\right) \Xi^{-1}-\pi\left(r_{2}^{2}+a^{2}\right) \Xi^{-1} .
$$

It is crucial to note that the action is independent of the time identification period $\Delta \tau$ and therefore, the manifold obtained is qualified as a constrained instanton. The relative probability of the Kerr-Newman black hole pair creation from the constrained instanton is

$$
P \approx \exp \left(\pi\left(r_{1}^{2}+a^{2}\right) \Xi^{-1}+\pi\left(r_{2}^{2}+a^{2}\right) \Xi^{-1}\right) .
$$

This is the exponential of one quarter of the sum of the outer and inner black hole horizon areas.

These two Fourier transformations are critical. Without them one cannot even obtain the constrained gravitational instanton. The inclusion of the extra term due to the Fourier transformation for the electrically charged rotating black hole pair also recovers the duality between the magnetic and electric cases [3][8][9][10].

The construction of the constrained instanton using the inner and outer black hole horizons is quite counter-intuitive. One could also consider those constructions involving other horizons as the instantons. However, the real part of the action for our choice is always greater than that of the other choices for the given configuration, and the wave function or the probability is determined by the classical orbit with the greatest real part of the action [1].

By the same argument, one has to use the pair of complex horizons $r_{0}, r_{1}$ to construct the constrained instanton for the case of open creation of black hole pair in the anti-de Sitter background. The relative probability of the Kerr-Newman black hole pair creation takes a form similar to (13) with a replacement of $r_{1}, r_{2}$ by $r_{0}, r_{1}$. One can show that the sum of the four horizon areas is $24 \pi / \Lambda$. Therefore, one can rewrite the relative probability as [8]

$$
P \approx \exp -\left(\pi\left(r_{2}^{2}+a^{2}\right) \Xi^{-1}+\pi\left(r_{3}^{2}+a^{2}\right) \Xi^{-1}\right) .
$$

This is the exponential of the negative of one quarter of the sum of the outer and inner black hole horizon areas.

It is interesting to note that the difference of relative probabilities in the closed and open creations of black hole pairs is the negative sign in the exponent. This is very reasonable from a physical argument. Since for both cases, the probability is a decreasing function of the mass parameter. This conclusion should be welcomed by quantum cosmologists. 
The case of the Kerr-Newman black hole family with spatially asymptotically flat infinity can be thought of as the limit of our case as we let $\Lambda$ approach 0 from below [8].

If one lets the angular momentum be zero, then it is reduced into the Reissner-Nordström(anti-)de Sitter black hole case. If one further lets the charge be zero, then it is reduced into the Schwarzschild-(anti-)de Sitter black hole case. There are only three horizons for the chargeless and nonrotating case.

For the Schwarzschild-de Sitter black hole case, one has to use the black hole and cosmological horizons to construct the instanton, the creation probability is the exponential of the entropy of the universe, or the exponential of one quarter of the sum of the black hole and cosmological horizon areas [3][11]. For the Schwarzschild-anti-de Sitter black hole case, one uses the pair of complex horizons to construct the instanton, and the creation probability is the exponential of the negative of the entropy. It is known that the entropy of the Schwarzschild-anti-de Sitter universe is one quarter of the black hole horizon area [12]. It is noted that the entropy is a decreasing(increasing) function of the mass parameter for the closed (open) model.

From the no-hair theorem, all stationary black holes in the de Sitter, anti-de Sitter and Minkowski spacetime backgrounds are described by these Kerr-Newman families, so the problem of black hole creations in these backgrounds is completely resolved. All known cases in the closed model are with regular instantons [9][10][11][13][14][15], and they can be considered as special cases of our study. The well known $S^{4}$ de Sitter model without black hole and $S^{2} \times S^{2}$ Nariai model with a pair of maximal black holes have the maximal and minimal creation probabilities, respectively [13].

Our treatment of quantum creation of the Kerr-Newman-anti-de Sitter space family using the constrained instanton can be thought of as a prototype of quantum gravity for an open system, without appealing to the background subtraction approach [16]. The beautiful aspect of our approach is that even in the absence of a general no-boundary proposal for open universes, we treat the creations of the closed and the open universes in the same way.

It can be shown that the probability of the universe creation without a black hole is greater than 
that with a pair of black holes in all these backgrounds.

\section{References:}

1. J.B. Hartle and S.W. Hawking, Phys. Rev. D료, 2960 (1983).

2. S.W. Hawking and N. Turok, Phys. Lett. B $\underline{425}, 25$ (1998), hep-th/9802030.

3. Z.C. Wu, Int. J. Mod. Phys. Dㅁ, 199 (1997), gr-qc/9801020.

4. Z.C. Wu, Gene. Relativ. Grav. 30, 1639 (1998), hep-th/9803121.

5. N. Turok and S.W. Hawking, Phys. Lett. B $\underline{432}, 271$ (1998), hep-th/9803156.

6. Z.C. Wu, Phys. Rev. D $\underline{31}, 3079$ (1985).

7. G.W. Gibbons and S.W. Hawking, Phys. Rev. D吕, 2738 (1977).

8. Z.C. Wu, Phys. Lett. B 445 , 274 (1999); gr-qc/9810077.

9. S.W. Hawking and S.F. Ross, Phys. Rev. D $\underline{52}, 5865$ (1995), hep-th/9504019.

10. R.B. Mann and S.F. Ross, Phys. Rev. D $\underline{52}, 2254$ (1995), gr-qc/9504015.

11. R. Bousso and S.W. Hawking, hep-th/9807148.

12. S.W. Hawking and D.N. Page, Commun. Math. Phys. 7, 577 (1983).

13. R. Bousso and S.W. Hawking, Phys. Rev. D $\underline{52}, 5659$ (1995), gr-qc/9506047.

14. F. Mellor and I. Moss, Phys. Lett. B $\underline{222}, 361$ (1989).

15. I.J. Romans, Nucl. Phys. B $\underline{383}, 395$ (1992).

16. S.W. Hawking, in General Relativity: An Einstein Centenary Survey, eds. S.W. Hawking and W. Israel, (Cambridge University Press, 1979). 